Submission ID: 43698

\title{
About Seismic Deconvolution and Some Opportunities of Results
}

Verification

D.B. Finikov* (Seismotech LLC)

\section{SUMMARY}

Opportunities of deconvolution results verification: problems of signal form estimation with the help of joint analysis of deconvolution results \& raw data are discussed. 
О задачах деконволюции сейсмических записей и возможностях контроля искажений формы сигнала.

\author{
Д.Б. Фиников (Яндекс Терра (ООО «Сейсмотек»))
}

\title{
Введение
}

Деконволюцию не любят обработчики. Деконволюцию не любят интерпретаторы. Однако без деконволюции не обходятся ни обработка, ни интерпретация. За что не любят деконволюцию? Эта процедура неизбежно поднимает шумы. Эта процедура непредсказуемым образом изменяет соотношения амплитуд. Эта процедура может порождать коррелированные артефактные события. Наконец, обоснования этой процедуры далеки от физики процессов распространения волн.

Когда выводятся основные соотношения для расчёта обратных фильтров, делаются предположения весьма произвольные, которые носят статистический характер, а представление о сейсмической записи, как о реализации случайного процесса с постулированными статистическими свойствами, вызывает законные сомнения в своей адекватности.

С другой стороны, деконволюция - единственный способ повысить разрешённость записи, разделить интерференционные события, повысить то, что геофизики называют динамической выразительностью волновой картины. Последнее понятие трудно формализуемо - в него входят и понятия разрешённости записи, и соотношения сигнал/шум, и так называемой «зарегулированности» волнового поля, и многие другие слова, которые легче произнести, чем формализовать, а тем более соотнести их с чем-то, что можно измерить. Поэтому тенденция в современной обработке с «сохранением истинных амплитуд» и динамических особенностей записи заключается в том, чтобы сделать процедуру обратной фильтрации как можно более «факторной». Что это значит?

Приоритет за деконволюциями, которые компенсируют влияние тех или иных физически обоснованных факторов, влияющих на форму сигнала. На суше - это поверхностносогласованная деконволюция, деконволюция, компенсирующая фактор поглощения, на море сигнатурная деконволюция, компенсация влияния газового пузыря, подавление волныспутника, при обработке вибросейсмических данных - специальные манипуляции с импульсом Клаудера. Можно перечислить еще факторы, за которые охотно производят коррекцию, считая их физически обоснованными. А всё, что не удаётся сделать этими средствами, оставляют на этап интерпретации, когда привлекают скважинную информацию и практикуют стратиграфическую деконволюцию, иногда скрытую в алгоритмах динамической инверсии. И после всего этого практически неизбежно применяются вполне заслуженные способы слепой деконволюции со всей их дурной репутацией. При этом стремление обойтись факторными деконволюциями зачастую приводит к тому, что используются ещё менее обоснованные алгоритмы, которые кажутся безобидными на сериях простых тестов, но могут создавать проблемы при формировании финальных изображений (простой пример с одноканальным антиспутником, включённым в сигнатурную деконволюцию - далеко не единственный).

В докладе делается попытка предложить концепцию решения деконволюционных задач с контролем преобразований динамических характеристик сигнала. Эта тема предваряется констатацией некоторых фактов, касающихся статистических основ деконволюции. Постановка задачи обратной фильтрации традиционно одноканальная, основанная на свёрточной модели трассы. При этом свёртка может быть заменена нестационарным линейным оператором. Многоканальность используется главным образом для вычисления необходимых статистических характеристик. 


\section{Теория}

Выпишем некоторые предположения и основные теоремные факты, касающиеся обобщённой свёрточной модели.

$$
Z(t)=P(t)+N(t)
$$

где $Z(t)$ - сейсмическая трасса, $P(t)$ - полезный сигнал, а $N(t)$ - аддитивная помеха некоррелированная с сигналом. Свойства помехи описывать не будем, поскольку рассмотрение обратной фильтрации при наличии помех, уведёт нас далеко от основной темы доклада.

$$
P(t)=g(t) \cdot\{q(t) * f(t)\},
$$

где $g(t)$ - функция, описывающая геометрическое расхождение, $f(t)$ - полосовой фильтр, ограничивающий диапазон частот значениями $F_{l}$ и $F_{h}$.

$$
q(t)=S(t) * \int d(t) L(\tau, t-\tau) d \tau,
$$

где $S(t)$ - форма импульса, включающая в себя кроме формы волны источника и другие свёрточные компоненты общие для всей трассы (например, влияние контакта земля/сейсмоприёмник, тракта записывающей аппаратуры и пр.), $d(t)$ - импульсная трасса (последовательность коэффициентов отражений). Про $L(\tau, t)$ полагают, что в диапазоне частот от $F_{l}$ до $F_{h}$ её преобразование Фурье описывается как

$$
\exp [-\rho(\tau) \cdot \tau \cdot(|w|+\mathrm{jC}(w))]
$$

где $\rho(\tau)$ - функция, которая определяется декрементом поглощения, $|w|$ и $C(w)$ связаны преобразованием Гильберта (минимально-фазовая модель поглощения). Основные статистические предположения касаются последовательности $d(t)$. Прежде всего, предполагают, что $d(t)$ - белый шум, т.е. $M[d(t) \cdot d(\tau)]=\sigma^{2} \cdot \delta(t-\tau)$, где $M-$ символ математического ожидания. Тогда в классе линейных преобразований с ядром $K(\tau, t)$, таким, что $K(\tau, \tau)=1, K(\tau, t)$ - минимально-фазовая функция по $t$ при каждом $\tau$ и среднее логарифма амплитудного спектра $K(\tau, t)$ на интервале от $F_{l}$ до $F_{h}$ равно 0, выполняется неравенство:

$$
M\left[\int \operatorname{dt}\left(\int P(\tau) K(\tau, t-\tau) d \tau\right)^{2}\right] \geq \sigma^{2} \int \operatorname{dt}(g(t) \cdot \exp (\phi(t)))^{2},
$$

где $\phi(t)$ целиком определяется функциями $\rho(\tau)$ и средним логарифма амплитудного спектра $S(t)$ в том же диапазоне частот. По функции $K(\tau, t)$ можно однозначно, при необременительных предположениях, восстановить амплитудный спектр сигнала $S(t)$ в диапазоне частот от $F_{l}$ до $F_{h}$ и функцию $\rho(\tau)$. Это неравенство обобщает результат, опубликованный в статье [1] и именно в таком виде использованный в работе [3]. Если эти величины известны, то форма импульса $S(t) * f(t)$ определяется с точностью до множителя и фазового спектра сигнала. В работах $[4,5]$ доказано, что, если $M\left(d^{4}(t)\right) \geq 3 \cdot M^{2}\left(d^{2}(t)\right)$ (процесс существенно негаусовский с положительным эксцессом), можно однозначно, с точностью до линейной компоненты определить и фазовый спектр сигнала (причём в работе [5] показано, как это делать по младшим дробным моментам записей).

Как можно понять из приведённых результатов, задача извлечения оценки формы сигнала из сейсмической записи разрешима, если возможно получить оценки соответствующих статистик. Однако, даже если статистические предположения верны, это большая проблема при имеющемся объёме данных. Но и при известной форме импульса задача деконволюции остаётся некорректной. Её можно ставить лишь в ограниченном диапазоне частот, где спектр сигнала существенно превалирует над спектром помехи. Следует также иметь в виду, что амплитуда сигнала в данной постановке не определяется. Полученные оценки всегда дают форму сигнала, средний логарифм спектра которого равен 0.

На этих фактах так или иначе основаны все способы обратной фильтрации стационарных и нестационарных сейсмических данных. Как было сказано, правомерность допущений о статистических свойствах импульсной трассы не вытекает ни из каких физических соображений, и на конкретных реализациях эти свойства не обязаны выполнятся (да и вообще, носят оценочный характер). Поэтому важно проверять устойчивость процедур по отношению к нарушению выполнения обозначенных предположений. Здесь становятся уместными 
различные подходы к реализации робастных методов деконволюции, но это не меняет существа обсуждаемой проблемы.

Некоторым исключением может казаться поверхностно-согласованная деконволюция. Она во многом наследует идеологию коррекции статических поправок и в этом смысле опирается на стационарную модель. Нелепо говорить о многооконной или переменной во времени поверхностно-согласованной деконволюции (хотя в обработке иногда и говорят о динамической статике, но это выглядит скорее курьёзом). Заметим, что на уровне статистик, которые используются в поверхностно-согласованной деконволюции, можно говорить, что она опирается на те же предположения, что и традиционная обратная фильтрация. Отличия в том, как эти статистики используются и разделяются на факторы. В задаче коррекции статических поправок давно осознали всю условность такой декомпозиции, её ненадежность и необходимость привлечения по возможности надёжной априорной информации для корректной постановки задачи. В задачах поверхностно-согласованной обработки динамических параметров этот аспект обычно стыдливо замалчивается, а всё, что относится к априорной информации о динамике, считают прерогативой интерпретаторов.

В докладе не приводятся новые алгоритмы обратной фильтрации, хотя они вытекают естественным образом из заявленных тезисов и, отчасти, реализованы. Акцент делается на возможностях контроля свёрточных преобразований сейсмических данных и связанных с ними методическими и алгоритмическими решениями.

Геофизики любят контролировать процедуры адаптивного подавления шума, разглядывая вычтенное поле помех и отыскивая в нём следы полезных сигналов. Иногда супервайзеры злоупотребляют этой техникой, доводя до абсурда стремление подавить помеху не затронув сигнал. Но в осмысленности такого рода контроля сомнений нет. В деконволюции такие средства контроля отсутствуют.

Речь пойдёт о подходе, отчасти сходном с ранее описанным способом эталонирования [2], применяемым при компенсации искажений миграционных преобразований.

Допустим, мы подвергли данные деконволюции. При этом, мы, конечно же, опирались на изложенные выше статистические предположения. Волновое поле обычно выглядит очень сложно, и качественные соображения о том, что мы сделали с формой сигнала, очень приблизительны. Можно теми или иными способами сгенерировать модельное поле, в котором сигналы свободны от интерференции. После этого можно посчитать свёрточный оператор, который переводит запись, подвергнутую обратной фильтрации в исходную запись. Оператор может быть и линейным нестационарным. Применим этот оператор к модельному полю (Рис. 1). Смысл этого фильтра в том, что он несёт в себе ту информацию о сигнале, на которой основывался расчёт обратного фильтра. Сопоставляя модельное поле и результат этого «матчинга» (жаргонное название формирующей фильтрации) мы можем видеть, что мы сделали с амплитудами и формой сигнала. Конечно, сам «матчинг», тоже основан на статистике и требует определённых методических и алгоритмических приёмов. Однако, это вполне контролируемая и понятная процедура, которая может стать необходимым элементом контроля в графе обработки. При этом специально для формирования такого эталона следует подбирать как параметры деконволюции, так и «матчинга». Например, параметры деконволюции могут (и должны) быть более жёсткими, чем обычно в обработке. Эти вопросы обсуждаются в докладе. 


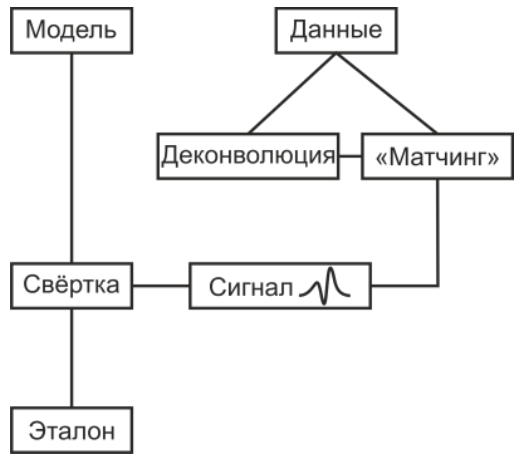

Рисунок 1 Схема формирования эталона

После того как такие эталоны сформированы, эта же техника может быть использована для контроля любой деконволюции уже с реальными параметрами, в том числе и поверхностносогласованных процедур.

Заметим, что как только искажения записи выявлены, немедленно следуют и алгоритмы коррекции этих искажений, что порождает новые алгоритмы деконволюции, но это обширная тема, выходящая за рамки данного сообщения.

Форма сигнала на формируемых эталонах определяется теми же предположениями, на которых основывались деконволюционные процедуры. Если сигнал предполагался минимально-фазовым или нуль-фазовым, мы такой и получим на эталоне. Если мы корректировали поглощение в некоторой модели, то и искажения сигнала будут обусловлены этой моделью. Если мы производили коррекцию фазового спектра, то эталону приписываются оценённые параметры фазы.

Как было отмечено, процедура требует моделирования отражённых волн от набора горизонтов. Понятно, что в этой задаче моделирование может быть совсем примитивным. Нуль-фазовые сигналы с равномерным спектром приписываются некоторым синфазностям, следующим друг за другом с заданным интервалом по временной шкале. На сейсмограммах им может быть приписана любая кинематика. Однако, для решения многих задач обработки и интерпретации моделирование - вполне содержательный и важный этап в графе преобразований сейсмических данных [2]. Описанная схема позволяет наполнить модельные поля динамикой, порождаемой реальными наблюдениями. Тогда и эталонные синфазности следует выбирать более содержательными, связанными с моделью среды. Процедура может оказаться полезной и при интерпретации мигрированных данных. В докладе приводятся примеры применения описанного подхода, иллюстрирующие возможности метода на модельных и реальных данных.

\section{Библиография}

1. Денисов М.С., Фиников Д.Б. Оценка относительного декремента поглощения сейсмических волн по критерию минимума дисперсии ошибки предсказания // Геология и геофизика. - 1999. - Т. 40, № 2. - С. 264-269.

2. Каплан С.А., Лебедев Е.Б., Шалашников А.В., Фиников Д.Б. Прямые задачи в обработке и интерпретации сейсмических данных // Конференция Санкт-Петербург 2016 «Через интеграцию геонаук к постижению гармонии недр», Санкт-Петербург 1114 апреля 2016 г.

3. Полубояринов М.А., Фиников Д.Б. Предсказывающая деконволюция в задаче коррекции нестационарных сейсмических записей // Технологии сейсморазведки. 2006. - № 1. - С. 26-36.

4. Фиников Д.Б., Сорин А.Я. Возможности определения формы сигнала в рамках традиционной модели сейсмической трассы. // Деп. ВИНИТИ. № 3625-84 деп.

5. Фиников Д.Б. Симметризующие фильтры // Технологии сейсморазведки. - 2007. -№ 1. - C. 26-36. 


\section{References}

1. Denisov M.S., Finikov D.B. Estimation of the relative decrement of seismic wave attenuation using the minimum criteria of dispersion error prediction // Geology and geophysics. - 1999. T.40, № 2. - C.264-269.

2. Kaplan S.A., Lebedev E.B., Shalashnikov A.V., Finikov D.B. Direct problem in the processing and interpretation of seismic data // Conference St. Petersburg 2016 "Through the integration of geoscience to understanding the harmony of the Earth's depths", St. Petersburg April 11-14, 2016.

3. Poluboyarinov M.A., Finikov D.B. Predicting deconvolution in the problem of correction of non-stationary seismic records // Technologies of seismic prospecting. - 2006. - No. 1. - P.2636.

4. Finikov D.B., Sorin A.Ya. Possibilities of the waveform evaluating within the traditional model of a seismic trace. // Dep. VINITI. No. 3625-84 dep.

5. Finikov D.B. Symmetrizing filters // Technologies of seismic prospecting. - 2007.-№1 .P.26-36. 